\title{
Unknown components of the plastidial permeome
}

\section{Thea R. Pick and Andreas P. M. Weber*}

Institut für Biochemie der Pflanzen, Cluster of Excellence on Plant Sciences, Heinrich-Heine Universität Düsseldorf, Düsseldorf, Germany

\author{
Edited by: \\ Ilka Haferkamp, University \\ Kaiserslautern, Germany \\ Reviewed by: \\ Karsten Fischer, University of Tromsø, \\ Norway \\ Hans-Henning Kunz, University of \\ California, San Diego, USA \\ *Correspondence: \\ Andreas P. M. Weber, Institut für \\ Biochemie der Pflanzen, Cluster of \\ Excellence on Plant Sciences, \\ Heinrich-Heine Universität Düsseldorf, \\ Universitätstrasse 1, D-40225 \\ Düsseldorf, Germany \\ e-mail: andreas.weber@uni- \\ duesseldorf.de
}

Beyond their role in photosynthesis plastids provide a plethora of additional metabolic functions to plant cells. For example, they harbor complete biosynthetic pathways for the de novo synthesis of carotenoids, fatty acids, and amino acids. Furthermore plastids contribute important reactions to multi-compartmentalized pathways, such as photorespiration or plant hormone syntheses, and they depend on the import of essential molecules that they cannot synthesize themselves, such as ascorbic acid. This causes a high traffic of metabolites across the plastid envelope. Although it was recently shown that non-polar substrates could be exchanged between the plastid and the ER without involving transporters, various essential transport processes are mediated by highly selective but still unknown metabolite transporters. This review focuses on selected components of the plastidial permeome that are predicted to exist but that have not yet been identified as molecular entities, such as the transporters for isopentenyl diphosphate (IPP) or ascorbic acid.

Keywords: chloroplast envelope membrane, metabolite transport, permeome

\section{INTRODUCTION}

Both, plastids and mitochondria are of endosymbiotic origin and play a pivotal role in energy supply and metabolism of plant cells. Both organelles harbor a plethora of metabolic processes and exchange a high amount of metabolites with the surrounding cell. Plastids are the site of photosynthesis, fatty acid synthesis, amino acid synthesis, isoprenoid synthesis, as well as sulfur and nitrogen assimilation. In addition, they contribute to pathways that are distributed across several compartments, such as photorespiration, pyrimidine synthesis, and hormone syntheses, leading to a high flux of metabolites over the plastid envelope.

The envelope of plastids of primary endosymbiotic origin consists of two distinct membranes, namely the outer (OE) and the inner envelope (IE) membranes. While the IE was understood as the de facto permeability barrier between cytosol and plastid, the $\mathrm{OE}$ was regarded as an unspecific membrane permeable for molecules smaller than $10 \mathrm{kDa}$. By now it is known that the OE is a metabolically active part of plastids (Schnurr et al., 2002) and contains pore forming proteins of different selectivity, like OEP16 (for outer envelope protein of $16 \mathrm{kDa}$; Pohlmeyer et al., 1997; Pudelski et al., 2012), OEP21 (Bolter et al., 1999), OEP24 (Pohlmeyer etal., 1998), and OEP37 (Goetze et al., 2006). Still it is controversial if the $\mathrm{OE}$ actively participates in the selective transport of metabolites (Flugge, 2000; Bolter and Soll, 2001).

Because a controlled exchange of metabolites across organellar boundaries is a pivotal requisite for proper function of plant metabolism the activity of specific transport proteins is indispensable. A prominent example for plastid envelope transporters is the triose phosphate/phosphate translocator (TPT; Flugge etal., 1989). The TPT belongs to the family of plastidic phosphate translocators and was the first transporter of the plastid envelope membrane that was identified at the molecular level and biochemically characterized in a heterologous system.
Recently it was shown that exchange of non-polar substrates between the plastid and the ER can proceed without the involvement of specific transport proteins by a membrane hemi-fusion mechanism (Mehrshahi et al., 2013). However, more than 100 putative plastidial transporters have been identified by in silico methods (Weber et al., 2005; Armbruster et al., 2011) and various chloroplast inner and outer envelope proteins that were detected by proteomics or transcriptomics are still awaiting functional characterization (Brautigam et al., 2008; Brautigam and Weber, 2009; Manandhar-Shrestha et al., 2013).

Hence, assuming that the abovementioned estimates are sensible, approximately $75 \%$ of the plastidial translocators remain unidentified as molecular entities. An example for the obstacles on the way to success is the recently identified photorespiratory glycolate glycerate transporter (Pick et al., 2013). The existence of this transport protein and its transport mode were established by biochemical assays already in the 1980s (Howitz and McCarty, 1985a,b, 1986). Nevertheless it took several decades to eventually link the biochemical function to a molecular entity, which became possible through multivariate statistical analyses of Arabidopsis gene expression datasets (Pick et al., 2013).

In this review we will focus on a subset of those unknown transporters, hence summarizing a selection of biochemical pathways with predicted metabolite transport steps over the plastid envelope (Figure 1).

\section{TRANSPORT OF ASCORBIC ACID}

In plants, ascorbate is present in all tissues and cell compartments (Zechmann etal., 2011). Up to four routes lead to the formation of ascorbate, including the L-galactose-pathway, the animal-like pathway via myo-inositol/glucuronate, the salvage pathway via L-galactonate, and the L-gulose-pathway. Among these four routes the biosynthetic route via L-galactose, also called the Smirnoff-Wheeler pathway, is the major pathway in many 


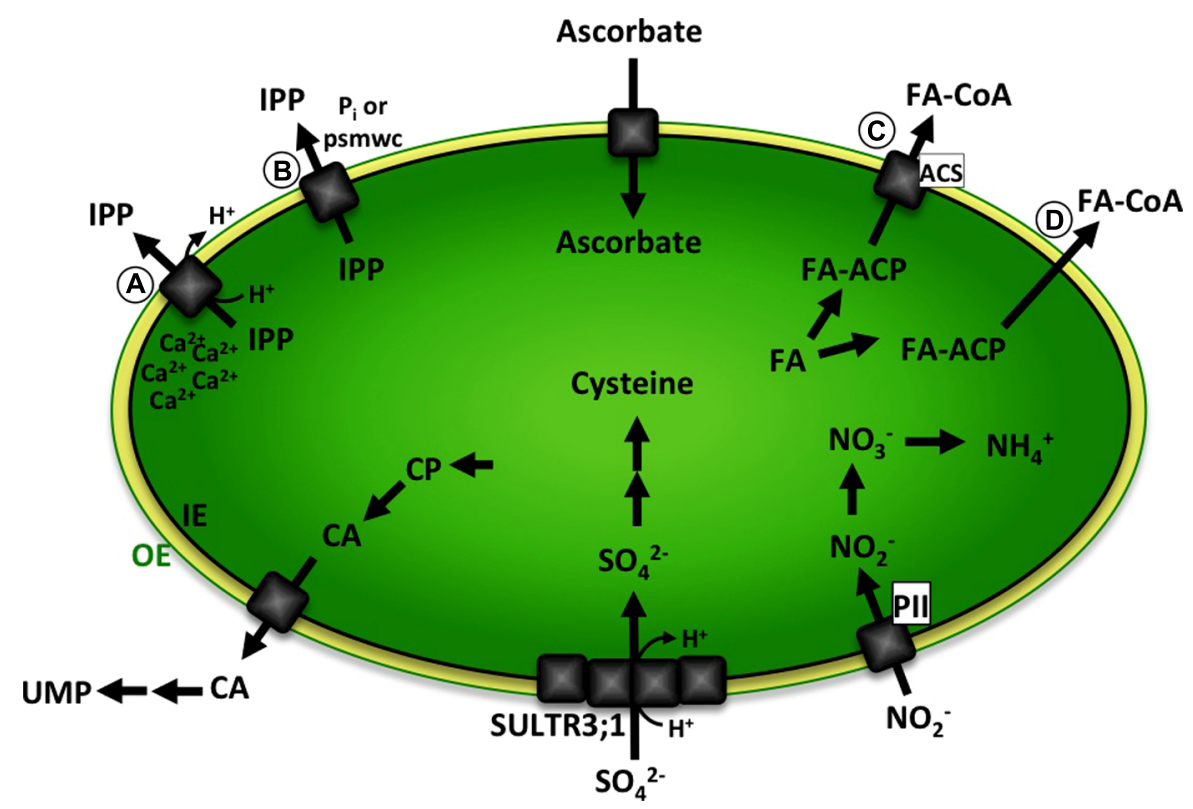

FIGURE 1 | Schematic representation of biochemical pathways with predicted metabolite transport steps over the plastid envelope discussed in this review. Ascorbate is exclusively produced in mitochondria and transported into plastids. IPP is either $(\mathbf{A})$ exported in a $\mathrm{Ca}^{2+}$-gated IPP/proton symport mechanism or (B) transport absolutely depends on binding of phosphorylated small molecular weight compounds (psmwc) to a regulatory side on the trans side of the membrane. Nitrite transport likely involves binding of plastidic PII protein. Sulfate is imported by a sulfate/proton cotransporter and is partially mediated by SULTR3;1, presumably involving other members of the SULTR3 family or a second independent transport system. CA is produced from CP in the plastid and exported to the cytosol. Further enzymatic steps in the cytosol and mitochondria lead to the formation of UMP. Fatty acids are synthesized in the plastid, attached to a ACP and exported either (C) involving a transport protein and binding of ACS in the outer envelope or (D) by facilitated diffusion leading to formation of FA-CoA. The green line represents the plastid outer envelope membrane (OE). The black line represents the plastid inner envelope membrane (IE). Black arrows indicate enzymatic steps. Gray squares represent transport proteins. OE, plastid outer envelope membrane; IE, plastid inner envelope membrane; psmwc, phosphorylated small molecular weight compounds; IPP, Isopentenyl diphosphate; PII, plastidic PII protein; CP, Carbamoyl phosphate; CA, Carbamoyl aspartate; UMP, uridine monophosphate; FA, free fatty acids; FA-ACP, fatty acid - Acyl carrier protein; ACS, acyl-coenzyme A (CoA) synthetase. plants (Wheeler et al., 1998; Gatzek et al., 2002; Laing et al., 2007; Bulley etal., 2009). In this pathway D-glucose is converted via the intermediates GDP-D-mannose and L-galactose to Lascorbate. Only recently the last missing enzyme in this pathway was identified, namely VTC2, a GDP-L-galactose phosphorylase (Laing et al., 2007). VTC2 was shown to be the rate-limiting step in ascorbate biosynthesis and overexpression of the VTC2 orthologous from kiwifruit (Actinidia spp.) in Arabidopsis resulted in an up to fourfold increase in ascorbate levels (Bulley et al., 2009). In addition, transient overexpression of VTC2 in Arabidopsis revealed its function as a major control point in light/dark regulation of ascorbate biosynthesis (Yoshimura et al., 2014). Except the last enzymatic step of ascorbate biosynthesis, the conversion of L-galactono-1,4-lactone to L-ascorbate by L-galactono-1,4-lactone dehydrogenase $(\mathrm{GLDH})$ on the inner mitochondrial membrane all enzymatic reactions take place in the cytosol. Among the other pathways the salvage pathway via L-galactonate was recently shown to essentially contribute to acsorbate levels in ripening tomato fruits (Badejo et al., 2012).

Since the last step of all four routes to ascorbate synthesis occurs on the inner mitochondrial membrane, transport into other organelles is essential (Horemans et al., 2000; Smirnoff and Wheeler, 2000). Moreover, ascorbate was shown to be distribute throughout the plant via the phloem from source to sink tissues (Franceschi and Tarlyn, 2002). Ascorbate is involved in many pathways, such as hormone biosynthesis, regenerating of other antioxidants, cell division and growth, and signal transduction. Furthermore, a recent study in pea and Arabidopsis embryos showed that ascorbate is crucial for iron uptake, as ascorbatemediated reduction is an obligatory step for the uptake of iron(II; Grillet et al., 2014). Ascorbate is essential for proper chloroplast function, since it acts as redox buffer in the Mehler peroxidase reaction with ascorbate peroxidase (APX) and thus regulates photosynthesis (Neubauer and Yamamoto, 1994; Caverzan et al., 2014). Hence, in photosynthetically active chloroplasts ascorbate concentration can reach up to $20 \mathrm{mM}$ (Foyer and Lelandais, 1996; Zechmann etal., 2011), which requires a high flux of this metabolite across the plastid envelope.

In plants, the best-characterized ascorbate transporter resides in the plasma membrane. It was characterized in isolated plasma membrane vesicles of Phaseolus vulgaris L., indicating an ascorbate/dehydroascorbate antiport mechanism (Horemans et al., 1996). Transport over the plastid envelope was determined biochemically. Uptake assays using intact isolated chloroplasts from Spinacia oleracea and Pisum sativum revealed that ascorbate is taken up by a saturable carrier that has a very low affinity $\left(K_{\mathrm{m}}=20 \mathrm{mM}\right)$, and does not transport glucose (Beck et al., 1983; Foyer and Lelandais, 1996). 
Recently Maurino etal. (2006) identified the nucleobaseascorbate transporter (NAT) gene family in Arabidopsis thaliana and rice (Oryza sativa) and Cai et al. (2014) in tomato (Solanum lycopersicum). Members of the NAT family share similarities with both transporters involved in uptake of nucleobases from fungi and the well characterized mammalian sodium-dependent ascorbate transporters (Gournas et al., 2008; Buerzle et al., 2013), making the members of the plant NAT family promising candidates for ascorbate transporters. Interestingly, AtNAT12 along with OsNAT10 and OsNAT11 were predicted to be plastid localized but subcellular localization of AtNAT12 indicate that it is localized to the plasma membrane (Maurino etal., 2006). Yet, ascorbate transport activity of plant NAT family members still remains to be demonstrated and the molecular nature of the plastid ascorbate transporter remains elusive.

\section{ISOPENTENYL DIPHOSPHATE (IPP)}

Isoprenoids are essential metabolites found in all organisms. In plants, isoprenoid-derived compounds such as carotenoids, tocopheroles, sterols, and terpenes fulfill numerous biochemical functions in pivotal processes, including photosynthesis, membrane and hormone synthesis, and plant defense. While the universal five-carbon precursor IPP is produced via the mevalonic acid (MVA) pathway in animals and fungi and the methylerythritol phosphate (MEP) pathway in many bacteria, both pathways occur, albeit in different compartments, in plants (Lange etal., 2000). Here, IPP synthesis from acetylCoA via the MEP pathway operates in the cytosol (McGarvey and Croteau, 1995), whereas IPP and its double bond isomer dimethylallyl diphosphate (DMAPP) are synthesized from triose phosphates and pyruvate in plastids via the 1-deoxy-Dxylulose 5-phosphate/methylerythritol phosphate (DOXP/MEP) pathway (Lichtenthaler et al., 1997). There is experimental evidence that both pathways cooperate in synthesis of specific metabolites (Adam et al., 1999). By inhibiting either the MEP or the MVA pathway, a metabolic crosstalk between both distinct pathways was shown, suggesting transporter mediated exchange of IPP between plastids and the cytosol (Laule etal., 2003). Also, a reduction of the import of the MEP pathway precursor pyruvate into chloroplasts in Arabidopsis mutant lines lacking the activity of the plastidial pyruvate:sodium symporter only led to a phenotype when the cytosolic MVA pathway was repressed in the mutant lines by application of the inhibitor mevastatin (Furumoto et al., 2011). However, the mode of IPP transport still remains controversial. Bick and Lange (2003) used intact chloroplasts and envelope membrane vesicles from Spinacia oleracea to identify the transport mode. They proposed a $\mathrm{Ca}^{2+}$-gated IPP/proton symport mechanism. On the other hand, Flugge and Gao (2005) showed that IPP transport over the plastid envelope proceeds via an uniport rather than an antiport mechanism and transport absolutely depends on binding of phosphorylated compounds to a regulatory side on the trans side of the membrane. However, to date none of the tested plastid envelope transporters mediates IPP transport, pointing to the existence of a yet unknown transporter for IPP.

\section{NITRITE AND SULFUR}

In plants primary ammonium $\left(\mathrm{NH}_{4}^{+}\right)$and sulfate assimilation takes place in chloroplasts. Nitrate $\left(\mathrm{NO}_{3}^{-}\right)$is taken up and reduced to nitrite $\left(\mathrm{NO}_{2}^{-}\right)$in the cytosol of mesophyll cells where nitrite is eventually taken up into chloroplast and further reduced to ammonium (Stitt et al., 2002). Uptake studies with chloroplast envelope membrane vesicles first pointed to rapid diffusion of nitrite, in the form of nitrous acid, across membranes without involvement of a transport protein (Shingles et al., 1996). However, uptake studies with intact chloroplasts revealed saturation kinetics that were highly comparable with those observed for nitrite reduction in chloroplasts, indicating a transporter mediated and regulated uptake of nitrite (Brunswick and Cresswell, 1988a,b). A nitrite transporter candidate, Nitrl-L, was shown to be chloroplast localized and Arabidopsis knockout mutants exhibited a five times higher accumulation of nitrite in leaves compared to wild type plants, pointing to a nitrite transport function of Nitr1-L (Sugiura et al., 2007). Moreover, it was shown that the plastidic PII protein is involved in nitrite uptake through a yet unknown mechanism (Ferrario-Mery et al., 2008). However, interaction of PII with Nitr1-L and biochemically direct nitrite uptake by Nitr1-L still remains to be demonstrated. In addition, a recent study localized Nitr1-L to the plasma membrane in grapevine and Arabidopsis, which makes it unlikely that this protein represents the plastidial nitrite transporter (Pike et al., 2014).

Recently AtNITR2;1, a transporter belonging to the HPP protein family, was identified as a new nitrite transporter candidate (Maeda et al., 2014). AtNITR2;1 is localized to the plastid IE (Ferro et al., 2003), expressed in shoot and root, and seems to be conserved in vascular plants (Maeda et al., 2014). When expressed in NA4, the nitrite transport-less mutant of the cyanobacterium Synechococcus elongatus AtNITR2;1 exhibited a high affinity for nitrite $\left(K_{\mathrm{m}}=13 \mu \mathrm{M}\right)$. Additionally, nitr2;1 Arabidopsis knockout mutants showed reduced uptake of nitrite into isolated chloroplasts compared to WT levels. From these experiments it was calculated that NITR2;1 accounts for $\sim 60 \%$ of nitrite uptake at $50 \mu \mathrm{M}$ external nitrite. However, since nitr2;1 mutant plants did not exhibit a visible phenotype when grown on nitrate as sole nitrogen source (Maeda etal., 2014), the physiological role of NITR2;1 still needs to be further investigated.

Sulfate is reduced and assimilated into cysteine and methionine in the chloroplast. Hence, sulfate has to be transported from the cytosol into chloroplasts. Transport studies revealed a sulfate import via a proton/sulfate co-transporter (Buchner et al., 2004). Recently a functional plastidic sulfate transporter SULTR3;1 was identified by knockout mutant analysis in Arabidopsis (Cao et al., 2013). SULTR3;1 belongs to the SULTR3 subfamily of plant sulfate transporters that is composed of five members (Takahashi et al., 2000). In yeast complementation studies SULTR3;1, SULTR3;2, and SULTR3;3 failed to complement the phenotype of the yeast mutant lacking both sulfur transporters (Takahashi et al., 2000), probably due to incorrect targeting of the proteins. However, in organello transport assays confirmed the proton/sulfate symport but revealed the existence of further plastidic sulfate transporters in addition to SULTR3;1 (Cao et al., 2013). In silico analysis revealed similarities in genomic organization of Sultr3;1 
and Sultr3;2 pointing to functional redundancy of SULTR3 subfamily members (Takahashi et al., 2000; Cao et al., 2013). Absence of strong phenotypes in single mutant plants additionally point to functional redundancy (Zuber et al., 2010). However, array data point to a low expression of SULTR3;2 throughout the plant (EFP browser Arabidopsis, Winter et al., 2007) implying the possibility of an additional transport system independent of SULTR3 family members. Further analysis of sultr3;1 sultr3;2 double and sultr3;1 sultr3;2 sultr3;3 sultr3;4 quadruple mutants may reveal how these transporters contribute to plastidic sulfate import.

\section{PYRIMIDINES}

Nucleotides, including purines and pyrimidines, are crucial components for plant primary and secondary metabolism and development. Pyrimidine de novo synthesis is a highly compartmentalized pathway involving enzymatic reactions in plastids, mitochondria, and the cytosol. The initial steps take place in plastids, eventually leading to the formation of carbamoyl aspartate (CA; Shibata etal., 1986; Chen and Slocum, 2008). In contrast to previous assumptions, where the conversion from CA to dihydroorotate (DHO) by DHOase took place in the plastids, protein-GFP fusion analysis could clearly localize the DHOase to the cytosol (Witz et al., 2012). This implies that CA has to be exported from plastids into the cytosol. In addition to de novo synthesis pyrimidines can also be synthesized via the less energy consuming salvage pathway. Presumably de novo synthesis in plants occurs in dividing cells and growing tissues where a high amount of nucleotides is required, whereas the salvage pathway operates in non-growing cells and mature tissues. Two studies revealed that the salvage pathway plays an essential role in the supply of pyrimidines to the plant (Mainguet et al., 2009; Chen and Thelen, 2011). Here it was shown that single mutant plants lacking plastidic uracil phosphoribosyltransferase (UPRT; Mainguet et al., 2009) and double mutant plants lacking both plastidic isoforms of uridine kinase (UKL1 and UKL2; Chen and Thelen, 2011) display dwarfish and chlorotic phenotypes.

Recently the plastidic uracil transporter PLUTO (plastidic nucleobase transporter) that is involved in the salvage pathway was identified (Witz et al., 2012). However, the transport mode of CA export during de novo synthesis and a transport protein remain unknown. As CA is a modified amino acid, transport through the DIT protein family, members of the plastidic amino acid permease (AAP) family, or the plant preprotein and amino acid transporter (PRAT) superfamily might be possible (Renne et al., 2003; Weber et al., 2005; Murcha et al., 2007).

\section{AMINO ACIDS}

Amino acids are the building blocks of enzymes and proteins and fulfill various additional functions in plants. They act as nitrogen donors for a variety of essential compounds and play an indispensable role in important processes like photorespiration. Plants are able to de novo synthesize all 20 proteogenic amino acids, while several enzymes involved in the biosynthesis of amino acids reside in the plastids and many amino acids are produced here (Kirk and Leech, 1972; Ravanel et al., 2004). Recently it was shown that the enzymatic steps of amino acid biosynthesis in plastids predominantly consist of non-cyanobacterial enzymes (Reyes-Prieto and Moustafa, 2012). Since protein synthesis occurs in the cytosol, plastids, and mitochondria, a high flux rate of amino acids across the plastid envelope is required, at least during developmental stages that are associated with high rates of protein biosynthesis. Hence, specific import and export proteins are required. To date, the only characterized plastidic IE amino acid transporter is AtDiT2.1 (Renne et al., 2003). AtDit2.1 mediates the exchange of glutamate/malate and also accepts the amino acid aspartate as transport substrate (Werner-Washburne and Keegstra, 1983, 1985). Based on several studies, in particular proteomics, transporter candidates for plastidic amino acid transporters were identified (Kleffmann etal., 2004; Brautigam et al., 2008). For example members of the PRAT superfamily PRAT1 and PRAT2 were shown to be plastid-localized (Murcha et al., 2007) but their transport function remains to be demonstrated.

\section{FATTY ACIDS}

In plants, plastids are the site of de novo fatty acid synthesis. Following synthesis, fatty acids are activated by a plastid localized acyl carrier protein (acyl-ACP) and are thus prepared for further complex lipid assembly (Ohlrogge et al., 1979). In Arabidopsis leaf mesophyll cells $62 \%$ of the chloroplastidic produced fatty acids are exported (Browse et al., 1986). In non-photosynthetic tissues and developing seeds of all plants even $90 \%$ of the fatty acids are exported (Browse et al., 1993). However, the molecular mechanism of fatty acid export from plastids is still controversial. It was proposed that membrane contact sites between plastids and endoplasmatic reticulum were involved (Andersson et al., 2007a,b) although experimental evidence is still missing. It has been shown, that before leaving the plastid, attaching the fatty acid to an acyl-ACP and fatty acid activation by acyl-coenzyme A (CoA) synthetase (ACS) forming fatty acyl-CoAs is mandatory (Ohlrogge et al., 2000). Both, acyl-ACP thioesterase and ACS were shown to be plastid localized with a clear localization of ACS to the plastid outer envelope (Andrews and Keegstra, 1983; Schnurr et al., 2002; Breuers et al., 2012). Recent rapid kinetic label experiments suggested that phosphatidylcholine participates in the export of newly synthesized acyl chains from plastids (Tjellstroem et al., 2012). But it is still under debate if fatty acid export is mediated by facilitated diffusion or specific transport proteins. The latter mechanism was already shown to occur in bacteria and yeast and involve activity of ACS proteins (Black and DiRusso, 2003). Recently it was also shown that the cyanobacterial ACS SLR1609 mediates the transport of fatty acids across a biological membrane (von Berlepsch et al., 2012). Transporter candidates in Arabidopsis were previously suggested but biochemical evidence for their involvement in fatty acid transport remains elusive (Koo and Ohlrogge, 2002).

\section{CONCLUSION}

Over the past decade substantial effort was invested in the identification of additional plastid envelope metabolite translocators as molecular entities. These recent studies led to the molecular identification of several transporters that were previously predicted 
on the basis of biochemical evidence, such as the plastidial pyruvate and glycolate/glycerate transporters (Furumoto et al., 2011; Pick et al., 2013). Recent "omics" and bioinformatics approaches, such as coexpression analysis, lead to the emergence of further candidates for putative transporters in the last years that, however, still await functional characterization (Brautigam et al., 2008; Brautigam and Weber, 2009; Majeran et al., 2010; Bordych et al., 2013; Manandhar-Shrestha et al., 2013).

\section{ACKNOWLEDGMENT}

We gratefully acknowledge continued support by the Deutsche Forschungsgemeinschaft (EXC 1028; IRTG 1525; FG 1186).

\section{REFERENCES}

Adam, K. P., Thiel, R., and Zapp, J. (1999). Incorporation of 1-1-C-13 deoxy-Dxylulose in chamomile sesquiterpenes. Arch. Biochem. Biophys. 369, 127-132. doi: 10.1006/abbi.1999.1346

Andersson, M. X., Goksor, M., and Sandelius, A. S. (2007a). Membrane contact sites: physical attachment between chloroplasts and endoplasmic reticulum revealed by optical manipulation. Plant Signal. Behav. 2, 185-187. doi: 10.4161/psb.2.3.3973

Andersson, M. X., Goksor, M., and Sandelius, A. S. (2007b). Optical manipulation reveals strong attracting forces at membrane contact sites between endoplasmic reticulum and chloroplasts. J. Biol. Chem. 282, 1170-1174. doi: 10.1074/jbc.M608124200

Andrews, J., and Keegstra, K. (1983). Acyl-CoA synthetase is located in the outer membrane and acyl-CoA thioesterase in the inner membrane of Pea chloroplast envelopes. Plant Physiol. 72, 735-740. doi: 10.1104/pp.72.3.735

Armbruster, U., Pesaresi, P., Pribil, M., Hertle, A., and Leister, D. (2011). Update on chloroplast research: new tools, new topics, and new trends. Mol. Plant 4, 1-16. doi: $10.1093 / \mathrm{mp} / \mathrm{ssq} 060$

Badejo, A. A., Wada, K., Gao, Y., Maruta, T., Sawa, Y., Shigeoka, S., et al. (2012). Translocation and the alternative D-galacturonate pathway contribute to increasing the ascorbate level in ripening tomato fruits together with the D-mannose/L-galactose pathway. J. Exp. Bot. 63, 229-239. doi: 10.1093/jxb/ err275

Beck, E., Burkert, A., and Hofmann, M. (1983). Uptake of L-ascorbate by intact spinach-chloroplasts. Plant Physiol. 73, 41-45. doi: 10.1104/pp.73.1.41

Bick, J. A., and Lange, B. M. (2003). Metabolic cross talk between cytosolic and plastidial pathways of isoprenoid biosynthesis: unidirectional transport of intermediates across the chloroplast envelope membrane. Arch. Biochem. Biophys. 415, 146-154. doi: 10.1016/s0003-9861(03)00233-9

Black, P. N., and DiRusso, C. C. (2003). Transmembrane movement of exogenous long-chain fatty acids: proteins, enzymes, and vectorial esterification. Microbiol. Mol. Biol. Rev. 67, 454-472. doi: 10.1128/mmbr.67.3.454-472.2003

Bolter, B., and Soll, J. (2001). Ion channels in the outer membranes of chloroplasts and mitochondria: open doors or regulated gates? EMBO J. 20, 935-940. doi: 10.1093/emboj/20.5.935

Bolter, B., Soll, J., Hill, K., Hemmler, R., and Wagner, R. (1999). A rectifying ATPregulated solute channel in the chloroplastic outer envelope from pea. EMBO J. 18, 5505-5516. doi: 10.1093/emboj/18.20.5505

Bordych, C., Eisenhut, M., Pick, T. R., Kuelahoglu, C., and Weber, A. P. M. (2013). Co-expression analysis as tool for the discovery of transport proteins in photorespiration. Plant Biol. 15, 686-693. doi: 10.1111/ plb. 12027

Brautigam, A., Hoffmann-Benning, S., and Weber, A. P. (2008). Comparative proteomics of chloroplast envelopes from $\mathrm{C} 3$ and $\mathrm{C} 4$ plants reveals specific adaptations of the plastid envelope to $\mathrm{C} 4$ photosynthesis and candidate proteins required for maintaining C4 metabolite fluxes. Plant Physiol. 148, 568-579. doi: 10.1104/pp.108.121012

Brautigam, A., and Weber, A. P. M. (2009). Proteomic analysis of the proplastid envelope membrane provides novel insights into small molecule and protein transport across proplastid membranes. Mol. Plant 2, 1247-1261. doi: 10.1093/mp/ssp070

Breuers, F. K. H., Brautigam, A., Geimer, S., Welzel, U. Y., Stefano, G., Renna, L., et al. (2012). Dynamic remodeling of the plastid envelope membranes - a tool for chloroplast envelope in vivo localizations. Front. Plant Sci. 3:7. doi: 10.3389/fpls.2012.00007
Browse, J., Mcconn, M., James, D., and Miquel, M. (1993). Mutants of Arabidopsis deficient in the synthesis of alpha-linolenate. Biochemical and genetic characterization of the endoplasmic nreticulum linoleoyl desaturase. J. Biol. Chem. 268, 16345-16351.

Browse, J., Warwick, N., Somerville, C. R., and Slack, C. R. (1986). Fluxes through the prokaryotic and eukaryotic pathways of lipid synthesis in the $16: 3^{\prime}$ plant Arabidopsis thaliana. Biochem. J. 235, 25-31.

Brunswick, P., and Cresswell, C. F. (1988a). Nitrite uptake into intact peachloroplasts.1. Kinetics and relationship with nitrite assimilation. Plant Physiol. 86, 378-383. doi: 10.1104/pp.86.2.378

Brunswick, P., and Cresswell, C. F. (1988b). Nitrite uptake into intact peachloroplasts. 2. Influence of electron-transport regulators, uncouplers, ATPase and anion uptake inhibitors and protein-binding reagents. Plant Physiol. 86, 384-389. doi: 10.1104/pp.86.2.384

Buchner, P., Stuiver, C. E. E., Westerman, S., Wirtz, M., Hell, R., Hawkesford, M. J., etal. (2004). Regulation of sulfate uptake and expression of sulfate transporter genes in Brassica oleracea as affected by atmospheric $\mathrm{H} 2 \mathrm{~S}$ and pedospheric sulfate nutrition. Plant Physiol. 136, 3396-3408. doi: 10.1104/pp.104. 046441

Buerzle, M., Suzuki, Y., Ackermann, D., Miyazaki, H., Maeda, N., Clemencon, B., et al. (2013). The sodium-dependent ascorbic acid transporter family SLC23. Mol. Aspects Med. 34, 436-454. doi: 10.1016/j.mam.2012.12.002

Bulley, S. M., Rassam, M., Hoser, D., Otto, W., Schuenemann, N., Wright, M., et al. (2009). Gene expression studies in kiwifruit and gene over-expression in Arabidopsis indicates that GDP-L-galactose guanyltransferase is a major control point of vitamin C biosynthesis. J. Exp. Bot. 60, 765-778. doi: 10.1093/jxb/ern327 Cai, X., Ye, J., Hu, T., Zhang, Y., Ye, Z., and Li, H. (2014). Genome-wide classification and expression analysis of nucleobase-ascorbate transporter (NAT) gene family in tomato. Plant Growth Regul. 73, 19-30. doi: 10.1007/s10725-013-9864-x

Cao, M.-J., Wang, Z., Wirtz, M., Hell, R., Oliver, D. J., and Xiang, C.-B. (2013). SULTR3;1 is a chloroplast-localized sulfate transporter in Arabidopsis thaliana. Plant J. 73, 607-616. doi: 10.1111/tpj.12059

Caverzan, A., Bonifacio, A., Carvalho, F. E. L., Andrade, C. M. B., Passaia, G., Schunemann, M., et al. (2014). The knockdown of chloroplastic ascorbate peroxidases reveals its regulatory role in the photosynthesis and protection under photo-oxidative stress in rice. Plant Sci. 214, 74-87. doi: 10.1016/j.plantsci.2013. 10.001

Chen, C. T., and Slocum, R. D. (2008). Expression and functional analysis of aspartate transcarbamoylase and role of de novo pyrimidine synthesis in regulation of growth and development in Arabidopsis. Plant Physiol. Biochem. 46, 150-159. doi: 10.1016/j.plaphy.2007.10.016

Chen, M., and Thelen, J. J. (2011). Plastid uridine salvage activity is required for photoassimilate allocation and partitioning in Arabidopsis. Plant Cell 23, 29913006. doi: 10.1105/tpc.111.085829

Ferrario-Mery, S., Meyer, C., and Hodges, M. (2008). Chloroplast nitrite uptake is enhanced in Arabidopsis PII mutant's. FEBS Lett. 582, 1061-1066. doi: 10.1016/j.febslet.2008.02.056

Ferro, M., Salvi, D., Brugiere, S., Miras, S., Kowalski, S., Louwagie, M., et al. (2003). Proteomics of the chloroplast envelope membranes from Arabidopsis thaliana. Mol. Cell. Proteomics 2, 325-345. doi: 10.1074/mcp.M300030MCP200

Flugge, U. I. (2000). Transport in and out of plastids: does the outer envelope membrane control the flow? Trends Plant Sci. 5, 135-137. doi: 10.1016/s13601385(00)01578-8

Flugge, U. I., Fischer, K., Gross, A., Sebald, W., Lottspeich, F., and Eckerskorn, C. (1989). The triose phosphate-3-phosphoglycerate phosphate translocator from spinach chloroplasts: nucleotide sequence of a full length cDNA clone and import of the in vitro synthesized precursor protein into chloroplasts. EMBO J. 8, 39-46.

Flugge, U. I., and Gao, W. (2005). Transport of isoprenoid intermediates across chloroplast envelope membranes. Plant Biol. 7, 91-97. doi: 10.1055/s-2004830446

Foyer, C. H., and Lelandais, M. (1996). A comparison of the relative rates of transport of ascorbate and glucose across the thylakoid, chloroplast and plasmalemma membranes of pea leaf mesophyll cells. J. Plant Physiol. 148, 391-398. doi: 10.1016/S0176-1617(96)80271-9

Franceschi, V. R., and Tarlyn, N. M. (2002). L-ascorbic acid is accumulated in source leaf phloem and transported to sink tissues in plants. Plant Physiol. 130, 649-656. doi: $10.1104 /$ pp.007062 
Furumoto, T., Yamaguchi, T., Ohshima-Ichie, Y., Nakamura, M., Tsuchida-Iwata, Y., Shimamura, M., etal. (2011). A plastidial sodium-dependent pyruvate transporter. Nature 478, 274-274. doi: 10.1038/nature10518

Gatzek, S., Wheeler, G. L., and Smirnoff, N. (2002). Antisense suppression of Lgalactose dehydrogenase in Arabidopsis thaliana provides evidence for its role in ascorbate synthesis and reveals light modulated L-galactose synthesis. Plant J. 30, 541-553. doi: 10.1046/j.1365-313X.2002.01315.x

Goetze, T. A., Philippar, K., Ilkavets, I., Soll, J., and Wagner, R. (2006). OEP37 is a new member of the chloroplast outer membrane ion channels. J. Biol. Chem. 281, 17989-17998. doi: 10.1074/jbc.M600700200

Gournas, C., Papageorgiou, I., and Diallinas, G. (2008). The nucleobase-ascorbate transporter (NAT) family: genomics, evolution, structure-function relationships and physiological role. Mol. Biosyst. 4, 404-416. doi: 10.1039/b719777b

Grillet, L., Ouerdane, L., Flis, P., Hoang, M. T. T., Isaure, M.-P., Lobinski, R., et al. (2014). Ascorbate efflux as a new strategy for iron reduction and transport in plants. J. Biol. Chem. 289, 2515-2525. doi: 10.1074/jbc.M113.514828

Horemans, N., Asard, H., and Caubergs, R. J. (1996). Transport of ascorbate into plasma membrane vesicles of Phaseolus vulgaris L. Protoplasma 194, 177-185. doi: $10.1007 / \mathrm{bf0} 1882025$

Horemans, N., Foyer, C. H., Potters, G., and Asard, H. (2000). Ascorbate function and associated transport systems in plants. Plant Physiol. Biochem. 38, 531-540. doi: 10.1016/s0981-9428(00)00782-8

Howitz, K. T., and McCarty, R. E. (1985a). Kinetic characteristics of the chloroplast envelope glycolate transporter. Biochemistry 24, 2645-2652. doi: 10.1021/bi00332a009

Howitz, K. T., and McCarty, R. E. (1985b). Substrate-specificity of the pea chloroplast glycolate transporter. Biochemistry 24, 3645-3650. doi: 10.1021/ bi00335a037

Howitz, K. T., and McCarty, R. E. (1986). D-glycerate transport by the pea chloroplast glycolate carrier - studies on [1-C-14] D-Glycerate uptake and D-glycerate dependent O-2 evolution. Plant Physiol. 80, 390-395. doi: 10.1104/pp.80.2.390

Kirk, P. R., and Leech, R. M. (1972). Amino acid biosynthesis by isolated chloroplasts during photosynthesis. Plant Physiol. 50, 228-234. doi: 10.1104/pp.50.2.228

Kleffmann, T., Russenberger, D., Von Zychlinski, A., Christopher, W., Sjolander, K., Gruissem, W., et al. (2004). The Arabidopsis thaliana chloroplast proteome reveals pathway abundance and novel protein functions. Curr. Biol. 14, 354-362. doi: 10.1016/j.cub.2004.02.039

Koo, A. J. K., and Ohlrogge, J. B. (2002). The predicted candidates of Arabidopsis plastid inner envelope membrane proteins and their expression profiles. Plant Physiol. 130, 823-836. doi: 10.1104/pp.008052

Laing, W. A., Wright, M. A., Cooney, J., and Bulley, S. M. (2007). The missing step of the L-galactose pathway of ascorbate biosynthesis in plants, an L-galactose guanyltransferase, increases leaf ascorbate content. Proc. Natl. Acad. Sci. U.S.A 104, 9534-9539. doi: 10.1073/pnas.0701625104

Lange, B. M., Rujan, T., Martin, W., and Croteau, R. (2000). Isoprenoid biosynthesis: the evolution of two ancient and distinct pathways across genomes. Proc. Natl. Acad. Sci. U.S.A. 97, 13172-13177. doi: 10.1073/pnas.240454797

Laule, O., Furholz, A., Chang, H. S., Zhu, T., Wang, X., Heifetz, P. B., et al. (2003) Crosstalk between cytosolic and plastidial pathways of isoprenoid biosynthesis in Arabidopsis thaliana. Proc. Natl. Acad. Sci. U.S.A. 100, 6866-6871. doi: 10.1073/pnas. 1031755100

Lichtenthaler, H. K., Schwender, J., Disch, A., and Rohmer, M. (1997). Biosynthesis of isoprenoids in higher plant chloroplasts proceeds via a mevalonate-independent pathway. FEBS Lett. 400, 271-274. doi: 10.1016/s00145793(96)01404-4

Maeda, S.-I., Konishi, M., Yanagisawa, S., and Omata, T. (2014). Nitrite transport activity of a novel HPP family protein conserved in cyanobacteria and chloroplasts. Plant Cell Physiol. 55, 1311-1324. doi: 10.1093/pcp/pcu075

Mainguet, S. E., Gakiere, B., Majira, A., Pelletier, S., Bringel, F., Guerard, F., et al. (2009). Uracil salvage is necessary for early Arabidopsis development. Plant J. 60, 280-291. doi: 10.1111/j.1365-313X.2009.03963.x

Majeran, W., Friso, G., Ponnala, L., Connolly, B., Huang, M., Reidel, E., et al. (2010). Structural and metabolic transitions of C4 leaf development and differentiation defined by microscopy and quantitative proteomics in maize. Plant Cell 22, 3509_ 3542. doi: 10.1105/tpc. 110.079764

Manandhar-Shrestha, K., Tamot, B., Pratt, E. P. S., Saitie, S., Braeutigam, A., Weber A. P. M., et al. (2013). Comparative proteomics of chloroplasts envelopes from bundle sheath and mesophyll chloroplasts reveals novel membrane proteins with a possible role in C4-related metabolite fluxes and development. Front. Plant Sci. 4:65. doi: 10.3389/fpls.2013.00065

Maurino, V. G., Grube, E., Zielinski, J., Schild, A., Fischer, K., and Fluegge, U.-I. (2006). Identification and expression analysis of twelve members of the nucleobase-ascorbate transporter (NAT) gene family in Arabidopsis thaliana. Plant Cell Physiol. 47, 1381-1393. doi: 10.1093/pcp/ pcl011

McGarvey, D. J., and Croteau, R. (1995). Terpenoid metabolism. Plant Cell 7, 10151026. doi: $10.1105 /$ tpc.7.7.1015

Mehrshahi, P., Stefano, G., Andaloro, J. M., Brandizzi, F., Froehlich, J. E., and Dellapenna, D. (2013). Transorganellar complementation redefines the biochemical continuity of endoplasmic reticulum and chloroplasts. Proc. Natl. Acad. Sci. U.S.A. 110, 12126-12131. doi: 10.1073/pnas.1306331110

Murcha, M. W., Elhafez, D., Lister, R., Tonti-Filippini, J., Baumgartner, M., Philippar, K., etal. (2007). Characterization of the preprotein and amino acid transporter gene family in Arabidopsis. Plant Physiol. 143, 199-212. doi: 10.1104/pp.106.090688

Neubauer, C., and Yamamoto, H. Y. (1994). Membrane barriers and Mehler-peroxidase reaction limit the ascorbate available for violaxanthin deepoxidase activity in intact chloroplasts. Photosyn. Res. 39, 137-147. doi: 10.1007/bf00029381

Ohlrogge, J. B., Kuhn, D. N., and Stumpf, P. K. (1979). Subcellular localization of acyl carrier protein in leaf protoplasts of Spinacia oleracea. Proc. Natl. Acad. Sci. U.S.A. 76, 1194-1198. doi: 10.1073/pnas.76.3.1194

Ohlrogge, J., Pollard, M., Bao, X., Focke, M., Girke, T., Ruuska, S., et al. (2000) Fatty acid synthesis: from $\mathrm{CO}_{2}$ to functional genomics. Biochem. Soc. Trans. 28, 567-574. doi: 10.1042/0300-5127:0280567

Pick, T. R., Braeutigam, A., Schulz, M. A., Obata, T., Fernie, A. R., and Weber, A. P. M. (2013). PLGG1, a plastidic glycolate glycerate transporter, is required for photorespiration and defines a unique class of metabolite transporters. Proc. Natl. Acad. Sci. U.S.A. 110, 3185-3190. doi: 10.1073/pnas.1215142110

Pike, S., Gao, F., Kim, M. J., Kim, S. H., Schachtman, D. P., and Gassmann, W. (2014). Members of the NPF3 transporter subfamily encode pathogen-inducible nitrate/nitrite transporters in grapevine and Arabidopsis. Plant Cell Physiol. 55, 162-170. doi: 10.1093/pcp/pct167

Pohlmeyer, K., Soll, J., Grimm, R., Hill, K., and Wagner, R. (1998). A highconductance solute channel in the chloroplastic outer envelope from pea. Plant Cell 10, 1207-1216. doi: 10.1105/tpc.10.7.1207

Pohlmeyer, K., Soll, J., Steinkamp, T., Hinnah, S., and Wagner, R. (1997). Isolation and characterization of an amino acid-selective channel protein present in the chloroplastic outer envelope membrane. Proc. Natl. Acad. Sci. U.S.A. 94, 95049509. doi: 10.1073/pnas.94.17.9504

Pudelski, B., Schock, A., Hoth, S., Radchuk, R., Weber, H., Hofmann, J., et al. (2012). The plastid outer envelope protein OEP16 affects metabolic fluxes during ABAcontrolled seed development and germination. J. Exp. Bot. 63, 1919-1936. doi: 10.1093/jxb/err375

Ravanel, S., Block, M. A., Rippert, P., Jabrin, S., Curien, G., Rebeille, F., et al. (2004). Methionine metabolism in plants - Chloroplasts are autonomous for de novo methionine synthesis and can import S-adenosylmethionine from the cytosol. J. Biol. Chem. 279, 22548-22557. doi: 10.1074/jbc. M313250200

Renne, P., Dressen, U., Hebbeker, U., Hille, D., Flugge, U. I., Westhoff, P., et al. (2003). The Arabidopsis mutant dct is deficient in the plastidic glutamate/malate translocator DiT2. Plant J. 35, 316-331. doi: 10.1046/j.1365-313X.2003. 01806.x

Reyes-Prieto, A., and Moustafa, A. (2012). Plastid-localized amino acid biosynthetic pathways of Plantae are predominantly composed of non-cyanobacterial enzymes. Sci. Rep. 2:955. doi: 10.1038/srep00955

Schnurr, J. A., Shockey, J. M., De Boer, G. J., and Browse, J. A. (2002). Fatty acid export from the chloroplast. molecular characterization of a major plastidial acyl-coenzyme A synthetase from Arabidopsis. Plant Physiol. 129, 1700-1709. doi: $10.1104 /$ pp.003251

Shibata, H., Ochiai, H., Sawa, Y., and Miyoshi, S. (1986). Localization of carbamoylphosphate synthetase and aspartate carbamoyltransferase in chloroplasts. Plant Physiol. 80, 126-129. doi: 10.1104/pp.80.1.126

Shingles, R., Roh, M. H., and Mccarty, R. E. (1996). Nitrite transport in chloroplast inner envelope vesicles.1. direct measurement of proton-linked transport. Plant Physiol. 112, 1375-1381. 
Smirnoff, N., and Wheeler, G. L. (2000). Ascorbic acid in plants: biosynthesis and function. Crit. Rev. Biochem. Mol. Biol. 35, 291-314. doi: 10.1080/10409230008984166

Stitt, M., Muller, C., Matt, P., Gibon, Y., Carillo, P., Morcuende, R., et al. (2002). Steps towards an integrated view of nitrogen metabolism. J. Exp. Bot. 53, 959-970. doi 10.1093/jexbot/53.370.959

Sugiura, M., Georgescu, M. N., and Takahashi, M. (2007). A nitrite transporter associated with nitrite uptake by higher plant chloroplasts. Plant Cell Physiol. 48 , 1022-1035. doi: 10.1093/pcp/pcm073

Takahashi, H., Watanabe-Takahashi, A., Smith, F. W., Blake-Kalff, M., Hawkesford, M. J., and Saito, K. (2000). The roles of three functional sulphate transporters involved in uptake and translocation of sulphate in Arabidopsis thaliana. Plant J. 23, 171-182. doi: 10.1046/j.1365-313x.2000.00768.x

Tjellstroem, H., Yang, Z., Allen, D. K., and Ohlrogge, J. B. (2012). Rapid kinetic labeling of Arabidopsis cell suspension cultures: implications for models of lipid export from plastids. Plant Physiol. 158, 601-611. doi: 10.1104/pp.111.186122

von Berlepsch, S., Kunz, H.-H., Brodesser, S., Fink, P., Marin, K., Fluegge, U.-I., et al. (2012). The acyl-acyl carrier protein synthetase from synechocystis sp PCC 6803 mediates fatty acid import. Plant Physiol. 159, 606-617. doi: $10.1104 /$ pp. 112.195263

Weber, A. P. M., Schwacke, R., and Flugge, U. I. (2005). Solute transporters of the plastid envelope membrane. Annu. Rev. Plant Biol. 56, 133-164. doi: 10.1146/annurev.arplant.56.032604.144228

Werner-Washburne, M., and Keegstra, K. (1983). L-Aspartate transport into pea chloroplasts. Plant Physiol. 72, 182-182. doi: 10.1104/pp.78.2.221

Werner-Washburne, M., and Keegstra, K. (1985). L-Aspartate transport into pea chloroplasts: kinetic and inhibitor evidence for multiple transport systems. Plant Physiol. 78, 221-227. doi: 10.1104/pp.78.2.221

Wheeler, G. L., Jones, M. A., and Smirnoff, N. (1998). The biosynthetic pathway of vitamin C in higher plants. Nature 393, 365-369. doi: 10.1038/30728

Winter, D., Vinegar, B., Nahal, H., Ammar, R., Wilson, G. V., and Provart, N. J. (2007). An "electronic fluorescent pictograph" browser for exploring and analyzing L-scale biological data sets. PLoS ONE 2:e718. doi: 10.1371/journal.pone.0000718
Witz, S., Jung, B., Fuerst, S., and Moehlmann, T. (2012). De novo pyrimidine nucleotide synthesis mainly occurs outside of plastids, but a previously undiscovered nucleobase importer provides substrates for the essential salvage pathway in Arabidopsis. Plant Cell 24, 1549-1559. doi: 10.1105/tpc.112. 096743

Yoshimura, K., Nakane, T., Kume, S., Shiomi, Y., Maruta, T., Ishikawa, T., et al. (2014). Transient expression analysis revealed the importance of VTC2 expression level in light/dark regulation of ascorbate biosynthesis in Arabidopsis. Biosci. Biotechnol. Biochem. 78, 60-66. doi: 10.1080/09168451.2014. 877831

Zechmann, B., Stumpe, M., and Mauch, F. (2011). Immunocytochemical determination of the subcellular distribution of ascorbate in plants. Planta 233, 1-12. doi: 10.1007/s00425-010-1275-x

Zuber, H., Davidian, J.-C., Aubert, G., Aime, D., Belghazi, M., Lugan, R., et al. (2010). The seed composition of Arabidopsis mutants for the group 3 sulfate transporters indicates a role in sulfate translocation within developing seeds. Plant Physiol. 154, 913-926. doi: 10.1104/pp.110.162123.

Conflict of Interest Statement: The authors declare that the research was conducted in the absence of any commercial or financial relationships that could be construed as a potential conflict of interest.

Received: 29 April 2014; accepted: 01 August 2014; published online: 19 August 2014. Citation: Pick TR and Weber APM (2014) Unknown components of the plastidial permeome. Front. Plant Sci. 5:410. doi: 10.3389/fpls.2014.00410

This article was submitted to Plant Traffic and Transport, a section of the journal Frontiers in Plant Science.

Copyright (C) 2014 Pick and Weber. This is an open-access article distributed under the terms of the Creative Commons Attribution License (CC BY). The use, distribution or reproduction in other forums is permitted, provided the original author(s) or licensor are credited and that the original publication in this journal is cited, in accordance with accepted academic practice. No use, distribution or reproduction is permitted which does not comply with these terms. 